\title{
TERMINLARNI TARJIMA QILISH MASALALARI
}

Geldiyeva Xursandoy

Alisher Navoiy nomidagi Toshkent davlat

o'zbek tili va adabiyoti universiteti

Tarjima nazaryasi va amaliyoti fakulteti

205-guruh talabasi

xursanoygeldiyeva@gmail.com

Manzura Abjalova

Alisher Navoiy nomidagi Toshkent davlat

o'zbek tili va adabiyoti universiteti

"Axborot texnologiyalari" kafedrasi dots.v.b., f.f.f.d. (PHD)

manzura ok@mail.ru

Annotatsiya. Terminlar ma'lum bir soha doirasida qo'llaniladigan so'zlar hisoblanib, ulardan faqat o'sha sohada foydalaniladi. Ba'zi terminlarni ikki yoki uch sohada ham ko'rishimiz mumkin, ammo ular har birida alohida ma'no kasb etadi. Terminlar ham neologizmlar kabi fan-texnika rivoji, iqtisodiy va moliyaviy taraqqiyot, informatsion texnalogiyalarning kundalik turmushda muhim vositalar sifatida xizmat qilishi natijasida ko'plab innovatsion ishlanmalarning yaratilishi orqali yildan yilga yangilanadi. Mazkur maqolada terminlarning ko'p ma'nolilik xususiyati, ularni tarjima qilish masalalari va to ' $\mathrm{g}$ 'ri qo'llay olish ko'nikmalarini shakllantirish haqida ma'lumotlar berildi.

Kalit so'zlar: terminalogiya, termin, soha, fan-texnika, ko'p ma'nolilik, leksima, sanoat taarmoqlari.

\section{PROBLEMS OF TRANSLATION OF TERMS}

Annotation. Terms are words that are used in a particular field and only used in that field. Some terms can be found in two or three areas, but each has a different meaning. As a result of the use of information technology as an important tool in everyday life, the terms, like neologisms, are updated through the development of science and technology, economic and financial development, and the creation of many innovative developments. This article provides information on the ambiguity of terms, how to translate them, and how to use them correctly.

Key words: Terminology, terms, field, science and technology, uncertainty, vocabulary, industry. 
Аннотация. Термины -это слова, которые используются в определенной области и используются только в этой области. Некоторые термины можно найти в двух или трех областях, но у каждого свое значение. Термины, как и неологизмы, обновляются из года в год в связи с развитием науки и технологий, экономическим и финансовым прогрессом и созданием множества инновационных разработок в результате использования информационных технологий в качестве важного инструмента в повседневной жизни. В этой статье содержится информация о неоднозначности терминов, о том, как их переводить и как правильно их использовать.

Ключевые слова: Терминология, термины, отрасль, наука $u$ технологии, неоднозначность, лексика, отрасли.

Termin - grekcha terminus so'zidan olingan bo'lib, chek-chegara degan ma'noni bildiriadi. U fan-texnika, qishloq xo'jaligi, san'at va madaniyat sohasiga xos so $^{6} \mathrm{z}$ hisoblanadi. Terminologiya - terminlar haqidagi ta'limot va terminlar majmui degan ma'nolarni anglatadi. Terminologiya masalalari hamisha tilshunoslikning dolzarb masalalaridan biri bo'lib kelgan. Chunki terminlarning sohalar lug'aviy qatlamlaridagi o'rni va vazifasini belgilash, tushunchaning mazmun-mohiyatini to ' ${ }^{6}$ 'ri anglash imkonini beradi. Terminologiyaga bag'ishlangan ishlarning barchasida $\mathrm{u}$ yoki bu sohaning muayyan tushunchalarini anglatadigan, definitsiyaga ega bo'Igan, asosan, nominativ funktsiyani bajaradigan birliklar termin hisoblanadi deb qaraladi [2].

Terminlar haqidagi fikrlarni olimlar nazaryasida ham ko'ra olamiz.

Masalan, A.Reformatskiy terminga ta'rif berar ekan, “... terminlar - bu maxsus so 'zlardir" degan xulosaga keladi.

A.V.Kalinin esa muayyan fanlar va kasbkorlikda ishlatiladigan so'zlarni "maxsus leksika" deb ataydi va uni ikki guruhga ajratadi.

1. Maxsus leksikaga, birinchi navbatda, terminlar kiradi.

2. Maxsus leksika tarkibiga terminlardan tashqari professionalizmlar ham kiradi.

U o'z fikrini davom ettirib, "Termin bilan professionalizmlar o'rtasidagi farq shuki, termin bu muayyan fan, sanoat sohasi, qishloq xo'jaligi, texnikadagi tamomila rasmiy bo'lgan, qabul qilingan va qonunlashtirilgan biror tushunchaning ifodasidir, professionalizm esa biror kasb, mutaxassislik, ko'pincha jonli tilda 
tarqalgan, aslini olganda, tushunchaning qat'iy, ilmiy tavsifiga ega bo'lmagan yarim rasmiy so 'zdir”,-deydi.

Olimlar qatorida H.Jamolxonov terminga munosabatda bo 'lar ekan quyidagicha yozadi: "Terminlar fan-texnika, adabiyot, san`at va boshqa sohlarga oid ixtisoslashgan, qo'llanishi muayyan soha bilan chegaralangan tushunchalarni ifodalaydigan nominativ birliklardir: gulkosa, shona (botanikada); to'rtburchak, kvadrat (geometriyada); ega, kesim (tilshunoslikda); qofiya, turoq, vazn (adabiyotshunoslikda) kabi ",-deya fikr bildiradi [1].

Bugungi kunda terminlar boshqa tillardan so'z olish va ichki so'z yasash hisobiga boyimoqda. $\mathrm{U}$ yoki bu soha terminologik tizimining barqarorligini belgilovchi asosiy omili uning tartibga solinganligi va muntazamligidir. Shu sababli ham terminalogiyasi ma'lum darajada barqarorlashgan barcha tillarda terminlar doimiy tartibga solib turiladi. Tartibga solish ma'lum terminologik me'yorlar asosida amalga oshiriladi.

Terminlar haqida keng ko'lamda gapirishdan avval, termin bilan oddiy so 'zning farqini tushunib olish zarur. Biz bu farqlanish haqida fikr bildirishdan oldin o'sha soha bilan shug'ullangan olimlarning nazaryalariga e'tibor qaratishimiz lozim. Ko'plab olimlar terminlarning quyidagi belgilarini sanab o'tishadi:

1) termin bir ma'noli tendentsiyaga ega;

2) termin aniq, nominativ funktsiyaga ega bo'lib, unga emotsionallik, ekspresivlik

funktsiyalari xos emas. Termin o'zining bu xususiyatini jumlalarda ham, matnlardan tashqarida ham saqlaydi;

3) terminning ma'nosi tushunchaga tengdir;

4) termin stilistik jihatdan neytraldir;

5) terminologik leksika alohida sistemadir [3];

Biz shuni bilib oldikki, yuqoridagi belgilarga qaragan holda oddiy so'z bilan terminning farqini ajrata olishimiz mumkin. Terminlarning yana bir xususiyati shundan iboratki, ular o'zi ishlatilinayotgan sohada maxsus termin sifatida, boshqa soha yoki tizimda oddiy so'z yoxud boshqa ma'noda ham qo'llaniladi.

Yuqoridagi fikrimning isboti o'laroq, oddiy so' $z$ va terminning farqi mavjud, ammo bir so 'z termin ham, oddiy so' $z$ ham bo'lib kelishi mumkin. Shu fikrlarimni tushuntirish uchun uch turga bo'lib ko'rsataman:

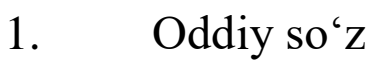

2. Termin 
3. Bir o'rinda oddiy so $\mathrm{z} z$, bir o'rinda termin

Oddiy so' $z$ - bu so'zlar doimo o'zining oddiy so'zligini saqlaydi. Termin bo'lib qo'llana olmaydi. Bularga atoqli otlar, olmosh va yordamchi so'zlar kiradi.

Termin - ular haqiqiy terminlardir, bular jumlalarda va undan tashqarida ham o'zinig terminalogik xususiyatini saqlab qoladi, oddiy so'z bilan qiyoslatilganda tubdan farq qiladi. Bunday so'zlarga tangenus, kotangenus, sinus, kosinus, affiks, suffiks, prefiks, sutemizuvchilar, umurtqasizlar kabilarni misol qilishimiz mumkin.

Bir o'rinda termin, bir o'rinda oddiy so' $z$ - bunday so'zlarni jumlalarda (kontekst) qo'llanilishiga qarab ajratish mumkin. Bunday so'zlar termin ham bo'lib kela oladi, oddiy so' $z$ o'rnida ham ishlata olishimiz mumkin. Misol o'rnida bo ' $\mathrm{g}$ 'in - tilshunoslikda termin, oddiy so'z o'rnida tana a'zosi, to'qima - biologiyada termin, oddiy so' $\mathrm{z}$ o'rnida to 'qilgan buyum (mn:jenfer), ildiz - biologiyada termin, oddiy so' $z$ o'rnida daraxt tomiri, kuch - fizikada termin (ot kuchi, kuchlanish), oddiy so'z o'rnida bilakdagi kuch va shunga o'xshash ko'plab so'zlarni aytishimiz mumkin.

Shu bilan birga, terminlarni oddiy so"zlardan tashqari kasb-hunarga doir so ‘zlardan ham farqlashimiz zarur. Kasb-hunarga oid so'zlar termin bo 'la olmaydi. Ular o'rtasida turli xil farqlar mavjud. Termin muayyan fan, ilm-texnika, qishloq xo'jaligi, sanoat va boshqa sohalarda rasmiy ravishda qo'llaniladigan, qonunlashtirilgan so 'zlar hisoblanadi. Kasb-hunarga oid so'zlar esa turli xil kasb va hunar turlariga doir so'zlar bo'lib, ular termin hisoblanmaydi. Masalan, poliz ekinlari, meva va taom nomlarini aytishimiz mumkin.

Terminlarning yildan yilga o'zgarishi va yangi terminlarning paydo bo'lishi fan va texnika taraqqiyoti bilan uzviy bog'liqdir. Tabiiy holda fan-texnika taraqqiyoti keng miqyosda yoyilayotganligi bois turli soha mutaxasislarining shu soha yutuqlaridan keng foydalanishi sababli terminlarga bo'lgan e'tibor ortmoqda va yangi terminlar paydo bo'lmoqda. Terminalogik nuqtai nazardan qaraydigan bo'lsak ham boshqa sohalarga nisbatan fan-texnikaga doir terminlar ko' $p$ va keng miqyosda foydalaniladi.

Boshqa xorijiy tillardagi turli sohalarga doir ilmiy matnlarni ya'ni terminlari mavjud bo'lgan matnlarni o'zbek tiliga tarjima qilish jarayonida yoki aksincha bo'lganda turli xil muommolar paydo bo'ladi. Ilk muommo va ayni paytda katta masalalardan biri qaysi tildan tarjima qilayotgan bo'lsangiz o'sha matndagi terminning ma'nosiga mos terminni narigi tildan tarjima qilishda topib bo'lmay qoladi. Bu katta qiyinchilik tug'diradi. Bundan avval kichik bir masalaga to'xtalib o'tsam, o'zbek tiliga boshqa tillardan (mn:ingliz tili) ilmiy ishlar tarjima 
qilinayotganda duch kelinayotgan terminlarni tarjima qilish uchun o'zbek tilida yaratilgan sohaviy terminlarga oid qo'llanmalar mavjud emas, bo'lsa ham to'liq emas, bu esa tarjimon va til o'rganuvchilari uchun terminlarni tarjima qilishda katta muommolarga sabab bo'ladi. O'ylashimcha yuqoridagi masalalar orqali bu uchinchi muommo paydo bo'lmoqda, bu muommo shuki, o'zga tildan termin tarjima qilinayotganda bizning tilimizda o'sha so'zga mosi topilmagandan so'ng o'sha chet so'zni o'z holicha (kalka) qabul qilishmoqda va o'sha so'zni tarjimasi orqali bizni tilda ko'radigan bo'lsak noto'g'ri ma'no bermoqda. Bu ham katta xato deb aytolamiz. Hozircha terminlarga oid bu masalalar yechilganicha yo 'q ammo, shunday muommolar mavjud ekan izlanish, o'rganish va bu masalalarni hal qilishga harakat qilish lozim.

Xulosa o'rnida aytish mumkinki, fan-texnika va barcha sohalar rivojlanar ekan terminlar ham ko'payadi va yangi terminlar vujudga keladi. Bu albatta yaxshi, ammo terminlarni vaqti - vaqti bilan saralab, tartiblab borish lozim. Shu bilan birga biz terminlar tarjimasi jarayonida vujudga kelayotgan muommolarni bartaraf etish uchun doimo o'rganish va harakatda bo'lishimiz lozim. Shundagina tarminalogiya rivojiga oz bo'lsa ham, o'z hissamizni qo'shgan bo'lamiz.

\section{Foydalanilgan adabiyotlar}

1. https://fayllar.org/til-va-terminologiya.html

2. https://azkurs.org/ajiniyoz-nomidagi-nukus-davlat-pedagogikainstituti.html?page $=2$

3. https://hozir.org/h-jamolxonov.html?page $=12$ 